\title{
Impact of Alkaline Pretreatment Condition on Enzymatic Hydrolysis of Sugarcane Bagasse and Pretreatment Cost
}

\section{Chaojun Wang}

Guangzhou Institute of Energy Conversion, CAS

\section{Wei Qi}

Guangzhou Institute of Energy Conversion, CAS

\section{Cuiyi Liang}

Guangzhou Institute of Energy Conversion, CAS

\section{Qiong Wang}

Guangzhou Institute of Energy Conversion, CAS

\section{Wen Wang ( $\nabla$ wangwen@ms.giec.ac.cn )}

Guangzhou Insititute of Energy Conversion, CAS https://orcid.org/0000-0002-3709-021X

\section{Zhongming Wang}

Guangzhou Institute of Energy Conversion, CAS

\section{Zhenhong Yuan}

Guangzhou Institute of Energy Conversion, CAS

\section{Original Article}

Keywords: Sodium hydroxide pretreatment, Combined severity factor, Lignocellulose, Biorefinery, Economic assessment

Posted Date: February 11th, 2021

DOI: https://doi.org/10.21203/rs.3.rs-182860/v1

License: (c) (1) This work is licensed under a Creative Commons Attribution 4.0 International License. Read Full License

Version of Record: A version of this preprint was published at Applied Biochemistry and Biotechnology on February 18th, 2021. See the published version at https://doi.org/10.1007/s12010-021-03530-y. 
Impact of alkaline pretreatment condition on enzymatic hydrolysis of sugarcane bagasse and pretreatment cost

Chaojun Wang, ${ }^{\mathrm{a}, \mathrm{b}}$, Wei Qi ${ }^{\mathrm{a}}$, Cuiyi Liang ${ }^{\mathrm{a}, \mathrm{b}}$, Qiong Wang ${ }^{\mathrm{a}}$, Wen Wang ${ }^{\mathrm{a},{ }^{*}}$, Zhongming

Wang $^{\mathrm{a}}$, Zhenhong Yuan ${ }^{\mathrm{a}, \mathrm{c}}$

${ }^{\text {a }}$ Guangzhou Institute of Energy Conversion, Chinese Academy of Sciences; CAS

Key Laboratory of Renewable Energy; Guangdong Provincial Key Laboratory of

New and Renewable Energy Research and Development, Guangzhou 510640, PR

China.

${ }^{\mathrm{b}}$ University of Chinese Academy of Sciences, Beijing 100049, PR China

${ }^{\mathrm{c}}$ Collaborative Innovation Centre of Biomass Energy, Zhengzhou 450002, PR China.

${ }^{*}$ Corresponding author.

Wen Wang, Email: wangwen@ms.giec.ac.cn; Tel.: (+86) 2037029690 


\section{Abstract}

A combined severity factor ( $\left.\mathrm{R}_{\mathrm{CSF}}\right)$ which is usually used to evaluate the effectiveness of hydrothermal pretreatment at above $100{ }^{\circ} \mathrm{C}$ had been developed to assess the influence of temperature, time and alkali loading on pretreatment and enzymatic hydrolysis of lignocellulose. It is not suitable for evaluating alkaline pretreatment effectiveness at lower than $100{ }^{\circ} \mathrm{C}$. According to the reported deducing process, this study modified the expression of $R_{C S F}=\log \left[C_{O H^{-}}^{n} \times t \times e^{\left(T_{r}-T_{b}\right) / 14.75}\right]$ as $R_{C S F}=$ $\log \left\{C_{\mathrm{OH}^{-}} \times t \times e^{[-13700 /(\operatorname{Tr}+273)+36.2]}\right\}$ which is easier and more reasonable to assess the effectiveness of alkaline pretreatment. It showed that $\mathrm{R}_{\mathrm{CSF}}$ exhibited linear trend with lignin removal, and quadratic curve relation with enzymatic hydrolysis efficiency (EHE) at the same temperature. The EHE of alkali-treated SCB could attain the maximum value at lower $\mathrm{R}_{\mathrm{CSF}}$, which indicated that it was not necessary to continuously enhance strength of alkaline pretreatment for improving EHE. Within a certain temperature range, the alkali loading was more important than temperature and time to influence pretreatment effectiveness and EHE. Furthermore, the contribution of temperature, time and alkali loading to pretreatment cost which was seldom concerned was investigated in this work. The alkali loading contributed more than $70 \%$ to the pretreatment cost. This study laid the foundation of further optimizing alkaline pretreatment to reduce cost for its practical application.

Keywords: Sodium hydroxide pretreatment; Combined severity factor; Lignocellulose; Biorefinery; Economic assessment

\section{Introduction}


Biorefinery of lignocellulosic biomass to biofuels and bioproducts has gained more and more attention due to its sustainability and low carbon discharge $(1,2)$. Lignocellulose is mainly composed of cellulose, hemicellulose and lignin which entangle with each other to endow the lignocellulose with recalcitrance towards biodegradation $(3,4)$. An ideal pretreatment is an indispensable step to break down the compact structure of lignocellulose for the following steps of enzymatic saccharification and microbial conversion $(3,5)$.

Various physical, chemical and biological tools have been adopted to pretreat lignocellulose like microwave, ultrasound, inorganic and organic solvents, microorganism, and so on. Pretreatment technologies including liquid hot water, diluted acid solution, steam explosion and diluted alkaline solution have been employed for pilot-scale biorefinery plant of lignocellulose. Different from other three pretreatment technologies which are conducted under the condition of above $100{ }^{\circ} \mathrm{C}$ and saturated steam pressure to mainly degrade hemicellulose ( 6 ), the alkaline pretreatment is more promising for industrial application due to its moderate condition which can be realized at lower temperatures than $100{ }^{\circ} \mathrm{C}$ without being pressurized and high lignin removal efficiency (7-9). $\mathrm{NaOH}$ is a common alkaline reagent to be used for pretreating lignocellulose due to its lower cost than potassium hydroxide $(\mathrm{KOH})$ and higher solubility than calcium hydroxide $\left(\mathrm{Ca}(\mathrm{OH})_{2}\right)$. However, the alkali-treated lignocellulose is often washed for several times to remove inhibitors and alkalis for guaranteeing high bioconversion efficiency, which would consume plenty of water and generate a large quantity of wastewater. The technologies 
including wastewater recycling (10) and direct bioconversion without washing step $(11,12)$ had been developed to overcome the obvious shortcomings of alkaline pretreatment process. Additionally, the attempt of recycling liquid waste from alkaline pretreatment as fertilizer (13) or something else would make alkaline pretreatment more feasible for actual application. Furthermore, to find out the main factors influencing pretreatment cost is another meaningful work for selecting proper and economical alkaline pretreatment condition for practical utilization. Based on the recycling technology of liquid wastes (13), the alkaline pretreatment cost investigated in this work is highly depended on energy consumption (relating to temperature and time), water consumption, and alkali loading. Seldom studies reported the factors influencing alkaline pretreatment cost with experimental tests.

As is known, the effectiveness of alkaline pretreatment is chiefly related to temperature, time and alkali loading. The combined severity factor $\left(\mathrm{R}_{\mathrm{CSF}}\right)$ which is modified from hydrothermal pretreatment $(14,15)$ had been used to describe the combined effects of temperature, time and alkali loading on alkaline pretreatment and enzymatic hydrolysis $(16,17)$.

$R_{C S F}=\log \left[C^{n} \times t \times \mathrm{e}^{\left(T_{r}-T_{b}\right) / 14.75}\right]$

where $R_{C S F}$ is the combined severity factor, $C$ is the concentration of hydroxyl ion $(\mathrm{mol} / \mathrm{L}), t$ is the reaction time $(\mathrm{min}), T_{r}$ is the temperature maintained during the reaction time $\left({ }^{\circ} \mathrm{C}\right), T_{b}$ is the base temperature $\left({ }^{\circ} \mathrm{C}\right)$ which is acknowledged as $100{ }^{\circ} \mathrm{C}$, and $n$ is an arbitrary constant. The expression of $\left(T_{r}-100\right) / 14.75$ where $T_{r}$ is in the range from 100 to $270{ }^{\circ} \mathrm{C}$ is equal to the equation of - 
$(13.7 \pm 0.3) \times\left[1000 /\left(T_{r}+273\right)\right]+(36.2 \pm 0.4)$ corresponding to the conventional first-order kinetics and Arrhenius model (18). As for the reaction happened below $100{ }^{\circ} \mathrm{C},\left(T_{r^{-}}\right.$ $100) / 14.75 \neq-(13.7 \pm 0.3) \times\left[1000 /\left(T_{r}+273\right)\right]+(36.2 \pm 0.4)$. The equation $(1)$ is deduced under the assumption that the reaction obeys to the first-order kinetics and the rate constant has relation to temperature via Arrhenius equation $(14,15,18)$. Therefore, in this study, the expression of $\left(T_{r}-100\right) / 14.75$ is replaced by $-13700 /\left(T_{r}+273\right)+36.2$ for alkaline pretreatment conducted below $100{ }^{\circ} \mathrm{C}$. The impact of alkaline pretreatment condition on lignin removal and enzymatic hydrolysis were switched to discuss the correlations between $R_{C S F}$ and lignin removal, and $R_{C S F}$ and enzymatic hydrolysis. The contribution of pretreatment condition on pretreatment cost was investigated in this study. The quota of temperature and time to pretreatment cost was reflected as energy consumption. At the similar enzymatic hydrolysis efficiency, the alkali loading shared more cost.

\section{Materials and methods}

\subsection{Materials}

Sugarcane bagasse (SCB) used in this experiment was collected from Guangxi Zhuang Autonomous Region (China). After being washed, it was dried to a constant weight in an oven at $105^{\circ} \mathrm{C}$, and then screened with 40-60 mesh sifter. After componential detection with the laboratory analytical procedure (NREL/TP-51042618) (19), the cellulose, hemicellulose and lignin contents of SCB were $43.98 \pm$ $0.57 \%, 23.62 \pm 0.36 \%$ and $23.61 \pm 3.84 \%$. The sodium hydroxide $(\mathrm{NaOH})$ was bought from Shanghai Macklin Biochemical Co., Ltd. (China). The cellulase was purchased 
from Imperial Jade Bio-technology Co., Ltd. (China). The filter paper activity of the cellulase was 197.5 FPU/g of powder, which was tested by Ghose's method (20).

\subsection{Alkali pretreatment}

The alkaline pretreatment was performed as the following procedure to evaluate the influence of temperature, time and $\mathrm{NaOH}$ loading on pretreatment effectiveness and cost. SCB was respectively added in $150 \mathrm{~mL}$ Erlenmeyer flasks containing $1 \%, 2 \%$, $3 \%, 4 \%(\mathrm{w} / \mathrm{v}) \mathrm{NaOH}$ solution to form solid-to-liquid ratio of $1: 10$. The flasks were placed in a water bath shaker with reciprocating rotation speed of $150 \mathrm{rpm}$ at $60^{\circ} \mathrm{C}$, $70{ }^{\circ} \mathrm{C}, 80^{\circ} \mathrm{C}$ for $1,2,3,4 \mathrm{~h}$, respectively. The electrical consumption of each pretreatment was recorded by metering socket. The treated SCB was washed with water to neutral $\mathrm{pH}$, and then oven-dried at $50{ }^{\circ} \mathrm{C}$ to a constant weight.

\subsection{Enzymatic hydrolysis}

The $\mathrm{NaOH}$-treated SCB was added into $25 \mathrm{~mL}$ Erlenmeyer flask containing $5 \mathrm{~mL}$, $0.05 \mathrm{M}$ acetate buffer ( $\mathrm{pH} 4.8)$ to achieve solid-to-liquid ratio of 1:10. The cellulase was loaded with $20 \mathrm{FPU} / \mathrm{g}$ cellulose. In order to prevent water loss, the flasks were sealed with glass plugs and parafilm in the hydrolysis process. The enzymatic hydrolysis was conducted at $50^{\circ} \mathrm{C}, 150 \mathrm{rpm}$ for $72 \mathrm{~h}$. Meanwhile, the electricity consumption during hydrolysis was also recorded by metering socket. And the enzymatic hydrolysis efficiency (EHE) was calculated by the followed formula:

$E H E \%=\frac{c_{\text {glucose }} \times v \times 0.9+c_{x y l o s e} \times v \times 0.88}{m_{\text {glucan }}+m_{x y l a n}} \times 100 \%$

where $c_{\text {glucose }}$ and $c_{x y l o s e}(\mathrm{mg} / \mathrm{mL})$ are the concentrations of glucose and xylose in the enzymatic hydrolysate, $v(\mathrm{~mL})$ is the volume of enzymatic hydrolysate, 0.9 and 0.88 
are the dehydration coefficients of the synthesis of glucose to glucan and xylose to xylan, respectively, and $m_{\text {glucan }}$ and $m_{x y l a n}(\mathrm{mg})$ are the mass of glucan and xylan in $\mathrm{NaOH}$-treated SCB.

\subsection{Calculation of the combined severity factor}

The combined severity factor (CSF) which describes the combined effect of temperature, time and alkaline concentration was analogized from the equation describing the combined effect of temperature, time and acid concentration for hydrothermal pretreatment $(18,21)$. It was modified as equation (1) to reflect the strength of alkaline pretreatment. The arbitrary constant $n$ in equation (1) is used to make the value of $R_{C S F}$ positive. Since $R_{C S F}$ is employed to exhibit the strength of pretreatment, it is not necessary to be positive. Based on the above description, it is finally modified as the following equation (3) with $n=1$.

$R_{C S F}=\log \left\{C_{O H^{-}} \times t \times e^{[-13700 /(T r+273)+36.2]}\right\}$

where $R_{C S F}, t$, and $T_{r}$ are the same as equation (1), and $\mathrm{COH}^{-}$is the concentration of hydroxyl ion (mol/L).

\section{Results and discussion}

\subsection{Correlation of the combined severity factor with $\mathrm{NaOH}$ pretreatment}

SCB was treated at 60,70 and $80^{\circ} \mathrm{C}$ with different $\mathrm{NaOH}$ concentrations for different times, respectively. The compositional changes of SCB and RCSF values after $\mathrm{NaOH}$ pretreatment were shown in Table 1. Under the condition of same temperature and $\mathrm{NaOH}$ loading, the relative contents of glucan (namely cellulose) and lignin tended to slightly increase and decrease, respectively. The relative xylan (namely hemicellulose) 
content changed little and then decreased slightly with $\mathrm{NaOH}$ loading surpassed $2 \%$ $(w / v)$. Under the condition of same temperature and time, the relative glucan content obviously showed increasing tendency, while the relative contents of xylan and lignin got the reverse trend with $\mathrm{NaOH}$ dosage increasing. Under the condition of same time and $\mathrm{NaOH}$ loading, the temperature could weakly influence the amount changes of glucan and xylan, but exhibit obvious impact on lignin removal. Generally, lignin removal is used as an indicator for evaluating the effectiveness of $\mathrm{NaOH}$ pretreatment under different conditions due to the chief action of alkaline pretreatment on lignin degradation $(7,22)$. The numeric size of $R_{C S F}$ value reflects the pretreatment strength which is resulted by temperature, time and alkali concentration during pretreatment. The same $\mathrm{R}_{\mathrm{CSF}}$ values appeared for $\mathrm{NaOH}$ pretreatment under the same temperature with different $\mathrm{NaOH}$ concentrations for different times (Table 1), which is in line with the previous reports $(16,17)$. The relationship between $\mathrm{R}_{\mathrm{CSF}}$ and lignin removal was shown in Fig. 1. The maximum lignin removal of $77.94 \%$ reached at $\mathrm{R}_{\mathrm{CSF}}=1.122(80$ ${ }^{\circ} \mathrm{C}, 3 \% \mathrm{NaOH}$ for $4 \mathrm{~h}$ ) has little difference with that of $77.40 \%$ obtained at $180{ }^{\circ} \mathrm{C}$, $10 \% \mathrm{NaOH} / \mathrm{Ethanol}$ for $30 \mathrm{~min}(23)$. RCSF tended to be linear correlation with the lignin removal, which is corresponding to the previous report (17). The $\mathrm{NaOH}$ concentration was more important for lignin removal than pretreatment time at the same RCSF such as sequential increment of lignin removal at $60{ }^{\circ} \mathrm{C}, 1 \% \mathrm{NaOH}$ for $4 \mathrm{~h}$, $60{ }^{\circ} \mathrm{C}, 2 \% \mathrm{NaOH}$ for $2 \mathrm{~h}$, and $60{ }^{\circ} \mathrm{C}, 4 \% \mathrm{NaOH}$ for $1 \mathrm{~h}$ (Table 1 and Fig. 1). The close RCSF values obtained under different temperatures, different $\mathrm{NaOH}$ loadings for different times showed that $\mathrm{NaOH}$ loading was at the first place to influence lignin 
removal, followed by temperature (Table 1 and Fig. 1). Additionally, the lignin removal has little difference when the pretreatment was conducted at $80^{\circ} \mathrm{C}$ with over $2 \% \mathrm{NaOH}$ for more than $2 \mathrm{~h}$. It indicated that lignin removal would not be improved further along with the intensification of alkaline pretreatment when it attained a limiting value.

\subsection{Correlation of the combined severity factor with enzymatic hydrolysis}

SCB treated under different conditions were enzymatically hydrolyzed at $50{ }^{\circ} \mathrm{C}$ for 72 $\mathrm{h}$ with addition of 20 FPU cellulase/g cellulose. From Fig. 2, the maximum EHE of SCB treated at $70{ }^{\circ} \mathrm{C}, 4 \% \mathrm{NaOH}$ for $4 \mathrm{~h}$ is $73.41 \%$ which is almost the same as those of SCB treated at $140 \sim 160{ }^{\circ} \mathrm{C}, 5 \% \mathrm{NaOH}$ mixed with $60 \%$ ethanol for 30 min (24). EHEs of SCB treated at same temperature for the same time were improved obviously with $\mathrm{NaOH}$ concentration increasing from $1 \%$ to $2 \%$, while had little difference when $\mathrm{NaOH}$ concentration was in the range from $2 \%$ to $4 \%$. Meanwhile, EHEs of SCB treated with over $2 \% \mathrm{NaOH}$ for the same time was changed little with pretreatment temperature increasing from $60{ }^{\circ} \mathrm{C}$ to $80{ }^{\circ} \mathrm{C}$. When $\mathrm{NaOH}$ loading achieved to $3 \%$, the EHEs of $\mathrm{NaOH}$-treated SCB had little differences no matter how different the pretreatment time and temperature were. It could be also found that EHEs of SCB treated with $2 \% \mathrm{NaOH}$ at $80{ }^{\circ} \mathrm{C}$ for $2 \mathrm{~h}, 3 \mathrm{~h}$ and $4 \mathrm{~h}$ had the maximum values around $70 \%$, as well as that of SCB treated with $3 \% \mathrm{NaOH}$ at $80{ }^{\circ} \mathrm{C}$ for $1 \mathrm{~h}$ (Fig. 2). As the pretreatment time prolonged for over $2 \mathrm{~h}$ and $\mathrm{NaOH}$ loading increased more than $2 \%$, EHEs of SCB treated at different temperatures were close to the maximum value. It meant that the optimum enzymatic hydrolysis of SCB treated with $\mathrm{NaOH}$ solution 
could be attained at lower energy consumption which is related to pretreatment temperature and time. The quadratic correlation between $\mathrm{R}_{\mathrm{CSF}}$ and EHE also showed that EHE had maximum value and would not be improved with R $\mathrm{CSF}$ increasing (Fig. 3). The EHE closed to the maximum value could be obtained at $60{ }^{\circ} \mathrm{C}, 3 \% \mathrm{NaOH}$ for $3 \mathrm{~h}$ whose $\mathrm{R}_{\mathrm{CSF}}$ was only higher than three conditions at $70{ }^{\circ} \mathrm{C}$, while lower than all conditions at $80{ }^{\circ} \mathrm{C}$. It hinted that a milder condition of alkaline pretreatment would realize maximum EHE of alkali-treated lignocellulose to reduce pretreatment cost.

\subsection{Contribution of temperature, time and alkali on pretreatment cost}

Under the same solid concentration and stirring speed, energy consumption and alkali loading are two main factors influencing pretreatment cost. Based on the previously constructed process that direct enzymatic hydrolysis of alkali-treated SCB without washing step (12), the three parameters - temperature, time and alkali loading, which are involved in the combined severity factor constitute the elements of alkaline pretreatment cost. The pretreatment conditions which made EHE of treated SCB 5\% less than the maximum EHE of SCB treated with $4 \% \mathrm{NaOH}$ at $70{ }^{\circ} \mathrm{C}$ for $4 \mathrm{~h}$ were selected to compare the cost. Through monitoring electricity, the costs of pretreating 1.6 kg SCB with solid-liquid ratio of 1:10 were estimated (Table 2). It could be found that the lowest pretreatment cost was attained under the condition of $2 \% \mathrm{NaOH}$ at 70 ${ }^{\circ} \mathrm{C}$ for $3 \mathrm{~h}$. Pretreatment conducted with more alkali loading at higher temperature for longer time got the higher cost, while the EHEs of treated SCB had little difference. The alkali consumption contributed a large portion of cost which ranged from $71.40 \%$ to $93.34 \%$. The pretreatment cost under the condition of $3 \% \mathrm{NaOH}$ at $60{ }^{\circ} \mathrm{C}$ for $3 \mathrm{~h}$ is 
higher than those costs under the conditions of $2 \% \mathrm{NaOH}$ at $70{ }^{\circ} \mathrm{C}$ for 3 and $4 \mathrm{~h}$, and $2 \% \mathrm{NaOH}$ at $80{ }^{\circ} \mathrm{C}$ for 2,3 and $4 \mathrm{~h}$. It hints that the reduction of alkali loading plays a more important role than energy consumption to reduce pretreatment cost when the EHEs of SCB treated under different conditions are close. Additionally, at the same alkali concentration, temperature has larger contribution on pretreatment cost than time under the circumstance of similar EHEs of treated SCB (Table 2). It indicates that a proper not a drastic alkaline pretreatment could realize desired EHE and reduce cost.

\section{Conclusion}

The combined severity factor $\left(\mathrm{R}_{\mathrm{CSF}}\right)$ inclines to present linear correlation with lignin removal and quadratic correlation with enzymatic hydrolysis efficiency. The alkali loading plays a more important role than temperature and time for lignin removal with the same and similar RCSF. The optimum enzymatic hydrolysis efficiency could be obtained for milder alkali-treated sugarcane bagasse. The alkali loading has larger contribution on pretreatment cost than energy consumption. The temperature acts a more obvious role than time for pretreatment cost under the same alkali loading.

\section{Declarations}

Funding. This work was financially supported by the National Key R\&D Program of China (2019YFB1503802), the Pearl River S\&T Nova Program of Guangzhou, China (201806010052), the National Natural Science Foundation of China (51861145103, 51976224, 52006227), Guangdong Basic and Applied Basic Research Foundation, China (2020A1515011012). 


\section{Conflicts of interest/Competing interests. None}

Ethical Approval. Not applicable

Consent to Participate. Not applicable

Consent to Publish. Not applicable

Availability of data and material. The data and material were obtained from experimental tests in duplicate and described clearly in the text.

Code availability. Not applicable

Authors Contributions. Conceptualization: Wen Wang, Wei Qi; Methodology:

Chaojun Wang, Cuiyi Liang; Formal analysis and investigation: Chaojun Wang;

Writing - original draft preparation: Chaojun Wang; Writing - review and editing:

Wen Wang, Wei Qi, Qiong Wang; Funding acquisition: Wei Qi, Wen Wang;

Resources: Cuiyi Liang, Qiong Wang; Supervision: Wen Wang, Zhongming Wang, Zhenhong Yuan.

\section{References}

1. Hassan, S.S., Williams, G.A., and Jaiswal, A.K. (2019). Moving towards the second generation of lignocellulosic biorefineries in the EU: Drivers, challenges, and opportunities. Renew. Sust. Energ. Rev. 101, 590-599.

2. Qi, W., Liu, G.F., He, C., Liu, S.N., Lu, S., Yue, J., Wang, Q., Wang, Z.M., Yuan, Z.H., and Hu, J.H. (2019). An efficient magnetic carbon-based solid acid treatment for corncob saccharification with high selectivity for xylose and enhanced enzymatic digestibility. Green Chem. 21(6), 1292-1304.

3. Liu, C.G., Xiao, Y., Xia, X.X., Zhao, X.Q., Peng, L., Srinophakun, P., and Bai, 
F.W. (2019). Cellulosic ethanol production: Progress, challenges and strategies for solutions. Biotechnol. Adv. 37(3), 491-504.

4. Lv, D., Xu, M., Liu, X., Zhan, Z., Li, Z., and Yao, H. (2010). Effect of cellulose, lignin, alkali and alkaline earth metallic species on biomass pyrolysis and gasification. Fuel Process. Technol. 91(8), 903-909.

5. Kumari, D., and Singh, R. (2018). Pretreatment of lignocellulosic wastes for biofuel production: A critical review. Renew. Sust. Energ. Rev. 90, 877-891.

6. Kucharska, K., Rybarczyk, P., Hołowacz, I., Łukajtis, R., Glinka, M., and Kamiński, M. (2018). Pretreatment of lignocellulosic materials as substrates for fermentation processes. Molecules. 23, 2937.

7. Kim, J.S., Lee, Y.Y., and Kim, T. H. (2016). A review on alkaline pretreatment technology for bioconversion of lignocellulosic biomass. Bioresour. Technol. 199, $42-48$.

8. Jung, W., Savithri, D., Sharma-Shivappa, R., and Kolar, P. (2018). Changes in lignin chemistry of switchgrass due to delignification by sodium hydroxide pretreatment. Energies. 11, 376.

9. Li, M., Fan, Y., Xu, F., Sun, R., and Zhang, X. (2010). Cold sodium hydroxide/urea based pretreatment of bamboo for bioethanol production: Characterization of the cellulose rich fraction. Ind. Crop. Prod. 32(3), 551-559.

10. Wang, W., Wang, Q., Tan, X.S., Qi, W., Yu, Q., Zhou, G.X., Zhuang, X.S., and Yuan, Z.H. (2016). High conversion of sugarcane bagasse into monosaccharides based on sodium hydroxide pretreatment at low water consumption and wastewater 
generation. Bioresour. Technol. 218, 1230-1236.

11. Wang, Q.F., Wang, W., Tan, X.S., Zahoor, Chen, X.Y., Guo, Y., Yu, Q., Yuan, Z. H., and Zhuang, X. S. (2019). Low-temperature sodium hydroxide pretreatment for ethanol production from sugarcane bagasse without washing process. Bioresour. Technol. 291, 121844.

12. Wang, Q., Wang, W., Zahoor, Tan, X., Zhuang, X., Miao, C., Guo, Y., Chen, X., Yu, Q., and Yuan, H. (2020). Recycling of black liquor for treating sugarcane bagasse at low temperature to attain high ethanol production without washing step. ACS Sustainable Chem. Eng. 8, 17016-17021.

13. Zahoor, Wang, W., Tan, X., Guo Y., Zhang, B., Chen, X., Yu, Q., Zhuang, X., and Yuan, Z. (2021). Mild Urea/KOH pretreatment to enhance enzymatic hydrolysis of corn stover with liquid waste recovery for plant growth. J. Clean. Prod. 284, 125392

14. Overend, R.P., and Chornet, E. (1987). Fractionation of lignocellulosics by steamaqueous pretreatments. Phil. Trans. R. Soc. Lond. (A), 321, 523-536.

15. Wyman, C.E., and Yang, B. (2017). Combined severity factor for predicting sugar recovery in acid-catalyzed pretreatment followed by enzymatic hydrolysis, In Ruiz H.A., Thomsen, M.H., Trajano, H.L. (eds.), Hydrothermal Processing in Biorefineries. Switzerland, Springer International Publishing AG, pp 161-180.

16. Júnior, A.D.N.F., Etchelet, M.I., Braga, A.F.M., Clavijo, L., Loaces, I., Noya, F., and Etchebehere, C. (2020). Alkaline pretreatment of yerba mate (Ilex paraguariensis) waste for unlocking low-cost cellulosic biofuel. Fuel. 266, 117068. 
17. Silverstein, R.A., Chen, Y., Sharma-Shivappa, R.R., Boyette, M. D., and Osborne, J. (2007). A comparison of chemical pretreatment methods for improving saccharification of cotton stalks. Bioresour. Technol. 98 (16), 3000-3011.

18. Chum, H.L., Johnson, D.K., and Black, S.K. (1990a). Organosolv pretreatment for enzymic hydrolysis of poplars. 2. Catalyst effects and the combined severity parameter. Ind. Eng. Chem. Res. 29(2), 156-162.

19. Sluiter, A., Hames, B., Ruiz, R., Scarlata, C., Sluiter, J., Templeton, D., and Crocker, D. (2008). Determination of structural carbohydrates and lignin in biomass. Technical Report NREL/TP-510-42618.

20. Ghose, T.K. (1987). Measurement of cellulase activities. Pure Appl. Chem. 59, 257-268.

21. Chum, H.L., Johnson, D.K., Black, S.K., and Overend, P. (1990b). Pretreatmentcatalyst effects and the combined severity parameter. Appl. Biochem. Biotechnol. $24 / 25,1-14$.

22. Wang, W., Wang, X., Zhang, Y., Yu, Q., Tan, X., Zhuang, X., and Yuan, Z. (2020). Effect of sodium hydroxide pretreatment on physicochemical changes and enzymatic hydrolysis of herbaceous and woody lignocelluloses. Ind. Crop. Prod. 145: 112145 .

23. Zhang, H., Zhang, J., Xie, J., Qin, Y. (2020). Effects of NaOH-catalyzed organosolv pretreatment and surfactant on the sugar production from sugarcane bagasse. Bioresour. Technol. 312, 123601.

24. Zhang, J., Xie, J., Zhang, H. (2021). Sodium hydroxide catalytic ethanol 
pretreatment and surfactant on the enzymatic saccharification of sugarcane bagasse.

Bioresour. Technol. 319, 124171. 
Fig. 1 Correlation between the combined severity factor ( $\left.\mathrm{R}_{\mathrm{CSF}}\right)$ and lignin removal, Exp is short for experimental data, Fitted is short for fitted data, and green line fits the whole data obtained under all conditions

Fig. 2 Enzymatic hydrolysis of SCB treated by $\mathrm{NaOH}$ solutions with different concentrations at $60{ }^{\circ} \mathrm{C}, 70^{\circ} \mathrm{C}$ and $80^{\circ} \mathrm{C}$ for different times, (a) $1 \mathrm{~h}$, (b) $2 \mathrm{~h}$, (c) $3 \mathrm{~h}$, (d) $4 \mathrm{~h}$. The enzymatic hydrolysis was conducted under the condition of solid-liquid ratio of $1: 10$, cellulase loading of $20 \mathrm{FPU} / \mathrm{g}$ cellulose, $150 \mathrm{rpm}, 50^{\circ} \mathrm{C}$ for $72 \mathrm{~h}$ Fig. 3 Correlation between the combined severity factor $\left(\mathrm{R}_{\mathrm{CSF}}\right)$ and enzymatic hydrolysis efficiency, Exp is short for experimental data, Fitted is short for fitted data, and green line fits the whole data obtained under all conditions 
Fig. 1

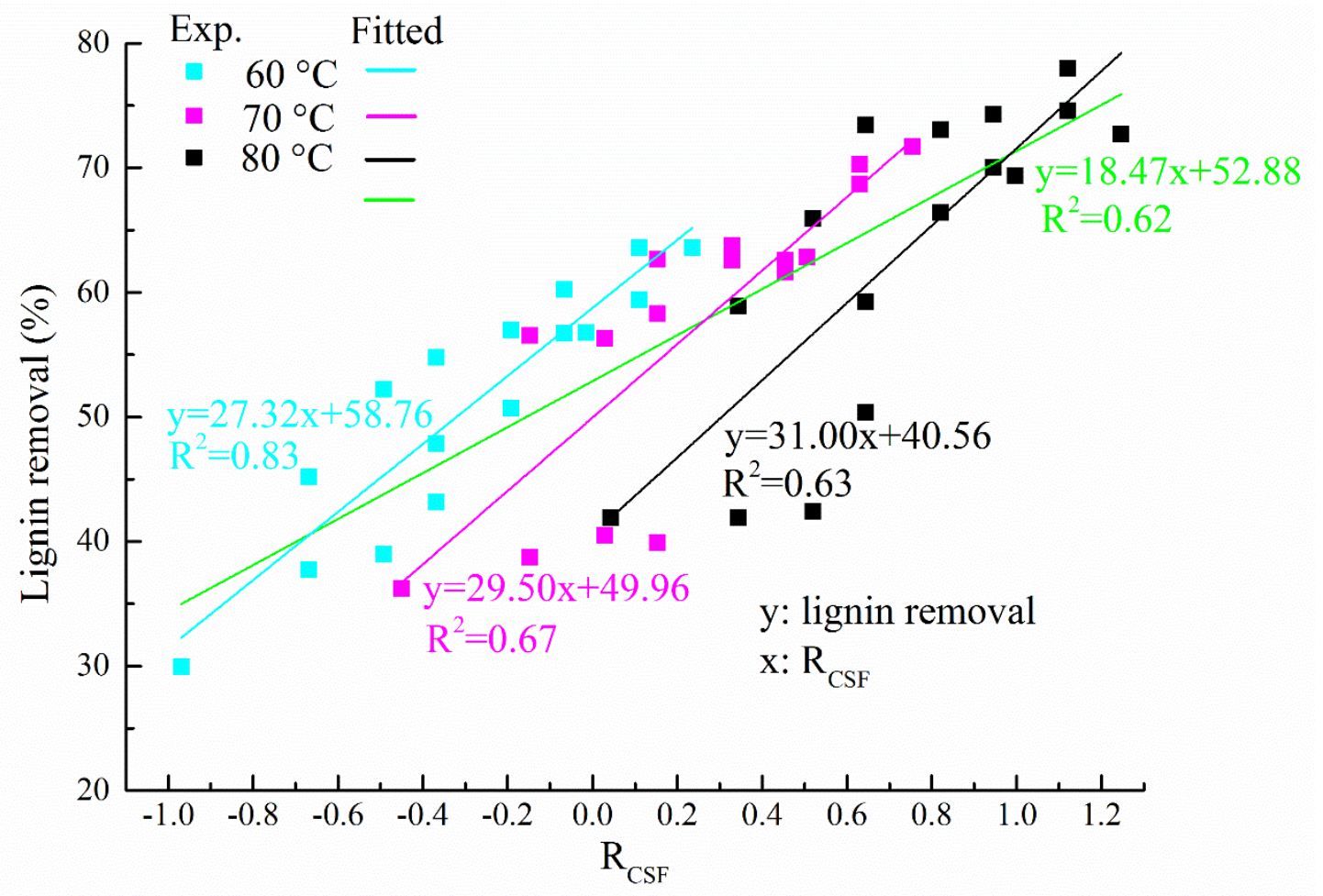


Fig. 2
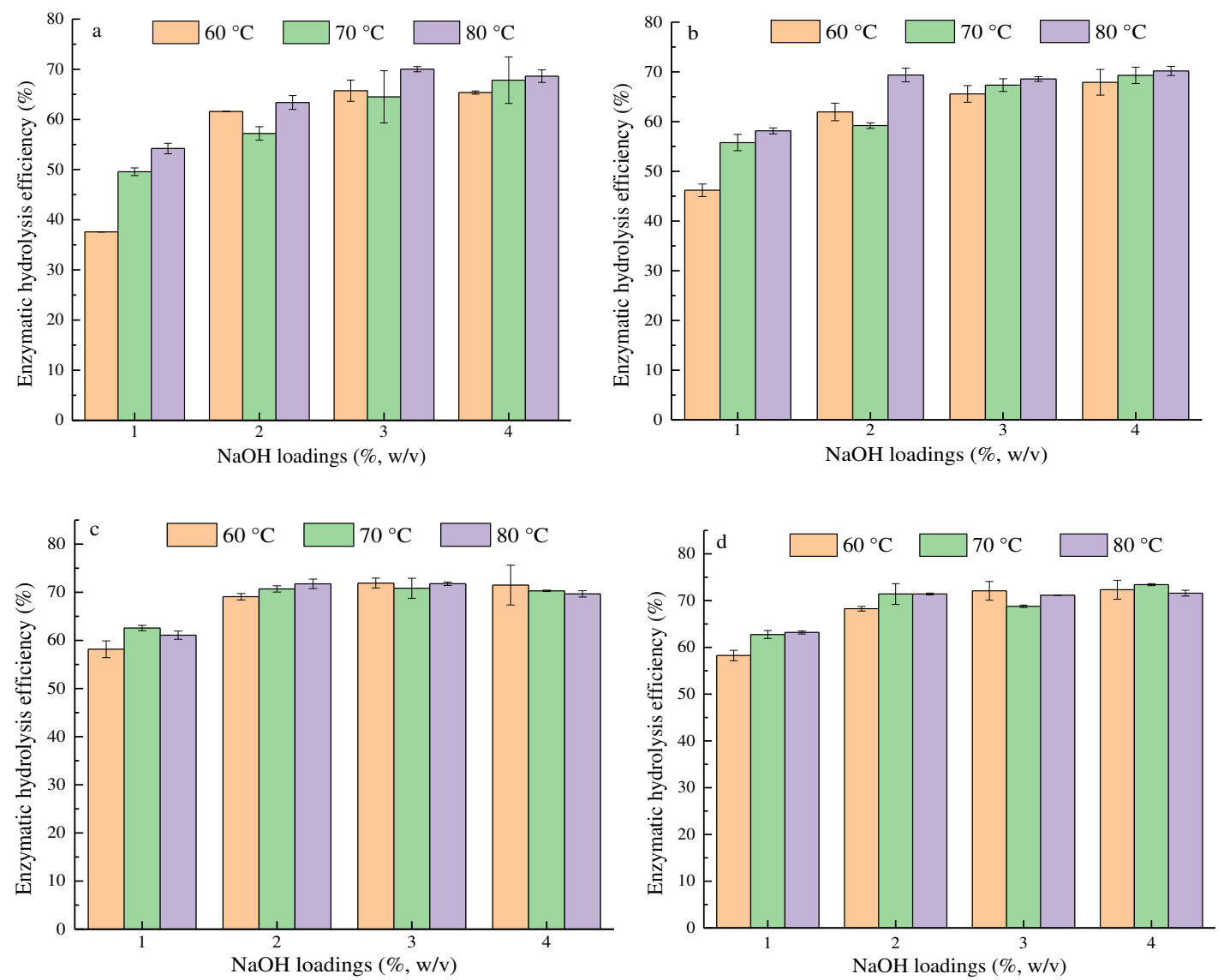
Fig. 3

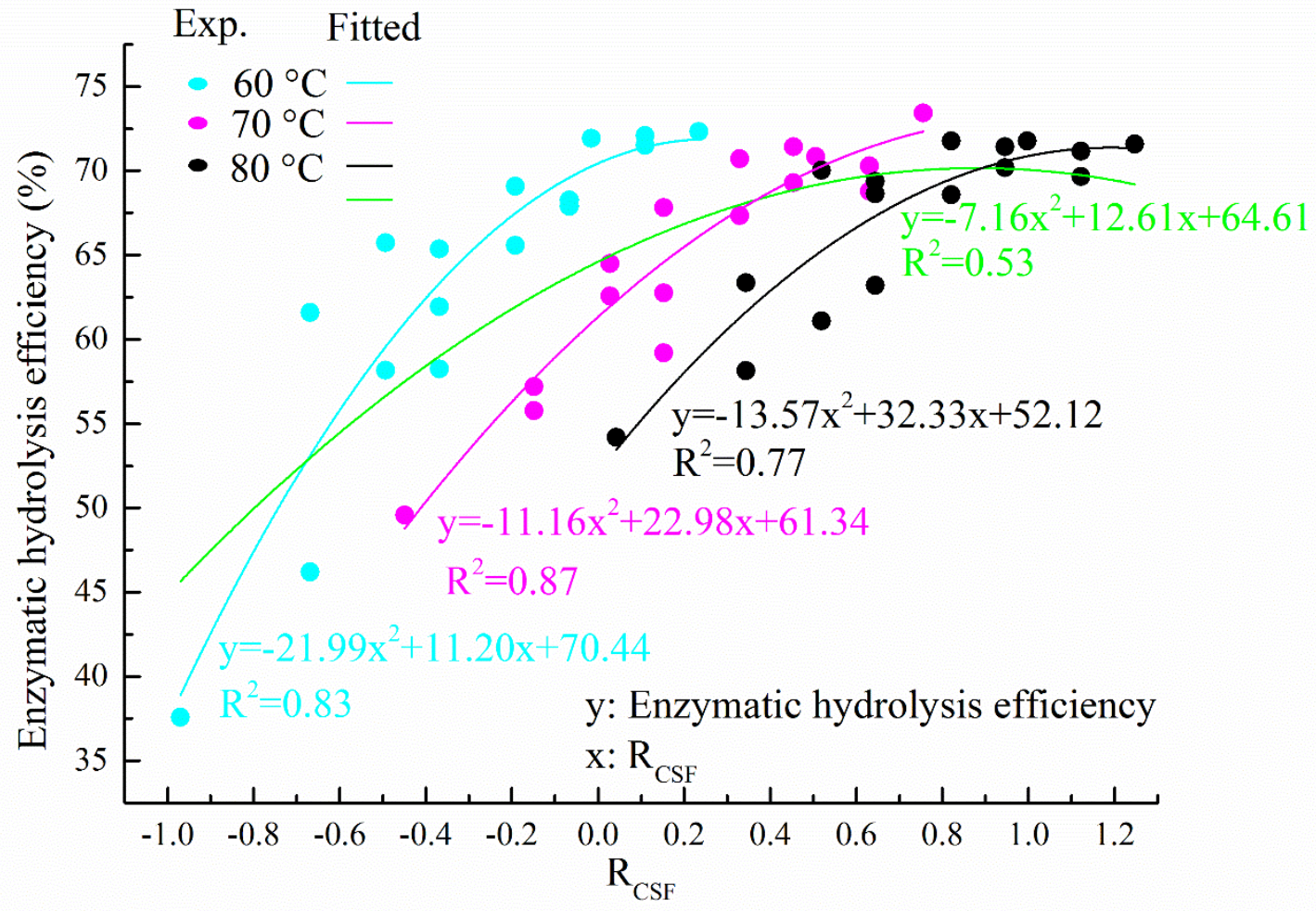


Table 1 Combined severity factor $\left(\mathrm{R}_{\mathrm{CSF}}\right)$ and composition of $\mathrm{NaOH}$-treated SCB

\begin{tabular}{|c|c|c|c|c|c|c|}
\hline $\mathrm{T}\left({ }^{\circ} \mathrm{C}\right)$ & $\begin{array}{l}\mathrm{NaOH} \text { conc. } \\
(\mathrm{g} / 100 \mathrm{~mL})\end{array}$ & $\mathrm{t}(\mathrm{h})$ & $\mathrm{R}_{\mathrm{CSF}}$ & Cellulose (\%) & Hemicellulose (\%) & Lignin $(\%)$ \\
\hline \multicolumn{2}{|c|}{ Raw material } & & & $43.98 \pm 0.571$ & $23.62 \pm 0.356$ & $23.61 \pm 3.84$ \\
\hline \multirow{16}{*}{60} & \multirow{4}{*}{1} & 1 & -0.970 & $48.29 \pm 0.32$ & $25.66 \pm 0.06$ & $19.30 \pm 1.00$ \\
\hline & & 2 & -0.669 & $50.69 \pm 0.02$ & $25.36 \pm 0.26$ & $17.46 \pm 4.85$ \\
\hline & & 3 & -0.493 & $51.63 \pm 0.95$ & $25.45 \pm 0.07$ & $17.26 \pm 2.11$ \\
\hline & & 4 & -0.368 & $52.54 \pm 0.07$ & $25.77 \pm 0.18$ & $16.64 \pm 0.70$ \\
\hline & \multirow{4}{*}{2} & 1 & -0.669 & $52.80 \pm 0.72$ & $23.34 \pm 0.48$ & $16.05 \pm 0.21$ \\
\hline & & 2 & -0.368 & $56.49 \pm 0.11$ & $24.39 \pm 0.11$ & $15.92 \pm 0.54$ \\
\hline & & 3 & -0.192 & $55.44 \pm 0.64$ & $23.90 \pm 0.14$ & $15.55 \pm 1.98$ \\
\hline & & 4 & -0.067 & $59.10 \pm 0.17$ & $23.79 \pm 0.04$ & $14.41 \pm 0.72$ \\
\hline & \multirow{4}{*}{3} & 1 & -0.493 & $55.39 \pm 0.41$ & $22.15 \pm 0.15$ & $15.55 \pm 0.17$ \\
\hline & & 2 & -0.192 & $61.14 \pm 0.16$ & $21.27 \pm 0.07$ & $14.85 \pm 0.82$ \\
\hline & & 3 & -0.016 & $62.06 \pm 0.12$ & $20.72 \pm 0.10$ & $14.77 \pm 2.89$ \\
\hline & & 4 & 0.109 & $62.84 \pm 0.14$ & $20.31 \pm 0.17$ & $14.39 \pm 1.66$ \\
\hline & \multirow{4}{*}{4} & 1 & -0.368 & $59.29 \pm 0.31$ & $20.70 \pm 0.11$ & $14.91 \pm 0.00$ \\
\hline & & 2 & -0.067 & $62.97 \pm 0.28$ & $19.23 \pm 0.22$ & $13.99 \pm 0.47$ \\
\hline & & 3 & 0.109 & $64.30 \pm 0.46$ & $18.62 \pm 0.10$ & $13.11 \pm 0.00$ \\
\hline & & 4 & 0.234 & $65.38 \pm 0.25$ & $18.08 \pm 0.13$ & $14.00 \pm 0.00$ \\
\hline \multirow{16}{*}{70} & \multirow{4}{*}{1} & 1 & -0.449 & $50.08 \pm 0.09$ & $25.79 \pm 0.00$ & $18.40 \pm 0.49$ \\
\hline & & 2 & -0.148 & $52.02 \pm 0.45$ & $26.86 \pm 0.09$ & $18.10 \pm 2.67$ \\
\hline & & 3 & 0.028 & $52.12 \pm 0.94$ & $26.58 \pm 0.16$ & $17.89 \pm 2.76$ \\
\hline & & 4 & 0.153 & $51.93 \pm 0.35$ & $26.35 \pm 0.29$ & $18.30 \pm 3.34$ \\
\hline & \multirow{4}{*}{2} & 1 & -0.148 & $54.40 \pm 0.59$ & $24.36 \pm 0.01$ & $13.55 \pm 0.95$ \\
\hline & & 2 & 0.153 & $56.44 \pm 0.67$ & $24.37 \pm 0.07$ & $13.69 \pm 0.23$ \\
\hline & & 3 & 0.329 & $56.83 \pm 0.17$ & $24.40 \pm 0.27$ & $12.70 \pm 0.17$ \\
\hline & & 4 & 0.454 & $57.54 \pm 0.12$ & $24.80 \pm 0.12$ & $13.35 \pm 1.30$ \\
\hline & \multirow{4}{*}{3} & 1 & 0.028 & $57.17 \pm 0.12$ & $22.15 \pm 0.15$ & $14.96 \pm 2.03$ \\
\hline & & 2 & 0.329 & $59.09 \pm 0.05$ & $21.33 \pm 0.02$ & $12.82 \pm 0.16$ \\
\hline & & 3 & 0.505 & $59.79 \pm 2.42$ & $20.01 \pm 0.62$ & $13.86 \pm 2.46$ \\
\hline & & 4 & 0.630 & $59.81 \pm 0.87$ & $19.36 \pm 0.52$ & $11.25 \pm 1.54$ \\
\hline & \multirow{4}{*}{4} & 1 & 0.153 & $59.02 \pm 1.13$ & $19.53 \pm 0.38$ & $13.23 \pm 0.52$ \\
\hline & & 2 & 0.454 & $60.95 \pm 0.15$ & $18.92 \pm 0.05$ & $13.93 \pm 2.87$ \\
\hline & & 3 & 0.630 & $62.82 \pm 0.49$ & $18.41 \pm 0.18$ & $11.98 \pm 0.35$ \\
\hline & & 4 & 0.755 & $63.50 \pm 0.27$ & $17.76 \pm 0.04$ & $11.12 \pm 1.00$ \\
\hline \multirow{7}{*}{80} & \multirow{4}{*}{1} & 1 & 0.042 & $50.76 \pm 1.04$ & $25.24 \pm 0.32$ & $17.43 \pm 0.00$ \\
\hline & & 2 & 0.344 & $52.70 \pm 0.42$ & $26.16 \pm 0.13$ & $15.46 \pm 2.27$ \\
\hline & & 3 & 0.520 & $53.27 \pm 0.33$ & $26.50 \pm 0.04$ & $17.99 \pm 0.16$ \\
\hline & & 4 & 0.645 & $53.87 \pm 0.04$ & $26.59 \pm 0.17$ & $15.58 \pm 1.47$ \\
\hline & \multirow{3}{*}{2} & 1 & 0.344 & $56.18 \pm 0.46$ & $23.81 \pm 0.20$ & $13.78 \pm 1.73$ \\
\hline & & 2 & 0.645 & $58.37 \pm 0.33$ & $23.75 \pm 0.24$ & $9.32 \pm 0.40$ \\
\hline & & 3 & 0.821 & $58.23 \pm 0.23$ & $23.70 \pm 0.37$ & $9.56 \pm 0.57$ \\
\hline
\end{tabular}




\begin{tabular}{llllll}
4 & 0.946 & $58.62 \pm 0.14$ & $23.94 \pm 0.32$ & $9.25 \pm 0.40$ \\
1 & 0.520 & $57.19 \pm 0.28$ & $20.82 \pm 0.07$ & $12.33 \pm 0.14$ \\
2 & 0.821 & $62.47 \pm 0.08$ & $20.76 \pm 0.20$ & $12.60 \pm 0.20$ \\
3 & 0.997 & $60.04 \pm 1.88$ & $20.57 \pm 0.08$ & $11.85 \pm 2.94$ \\
& 4 & 1.122 & $64.22 \pm 0.10$ & $20.40 \pm 0.18$ & $8.69 \pm 0.24$ \\
& 1 & 0.645 & $61.84 \pm 0.49$ & $18.79 \pm 0.33$ & $15.27 \pm 0.17$ \\
& 2 & 0.946 & $63.89 \pm 1.00$ & $18.02 \pm 0.12$ & $11.84 \pm 2.06$ \\
& 3 & 1.122 & $65.22 \pm 0.31$ & $19.19 \pm 1.19$ & $10.29 \pm 0.64$ \\
& 4 & 1.247 & $64.63 \pm 0.33$ & $17.70 \pm 0.08$ & $11.01 \pm 0.42$ \\
\hline
\end{tabular}


Table 2. Comparison of pretreatment cost with similar enzymatic hydrolysis

efficiency under different pretreatment conditions ${ }^{\mathrm{a}}$

\begin{tabular}{|c|c|c|c|c|c|c|c|c|}
\hline \multirow[b]{2}{*}{$\mathrm{T}\left({ }^{\circ} \mathrm{C}\right)$} & \multirow{2}{*}{$\begin{array}{c}\mathrm{NaOH} \text { conc. } \\
(\mathrm{g} / 100 \mathrm{~mL})\end{array}$} & \multirow[b]{2}{*}{$\mathrm{t}(\mathrm{h})$} & \multirow[b]{2}{*}{ EHE (\%) } & \multicolumn{2}{|c|}{ Electricity } & \multicolumn{2}{|c|}{$\mathrm{NaOH}$ loading } & \multirow{2}{*}{$\begin{array}{l}\text { Total } \\
\text { (US } \\
\text { dollar) }\end{array}$} \\
\hline & & & & $\mathrm{kWh}$ & $\begin{array}{l}\text { US } \\
\text { dollar }\end{array}$ & $\mathrm{g}$ & $\begin{array}{l}\text { US } \\
\text { dollar }\end{array}$ & \\
\hline \multirow{4}{*}{60} & \multirow{2}{*}{3} & 3 & $71.91 \pm 1.03$ & 2.19 & 0.20 & \multirow{2}{*}{480} & 2.20 & 2.41 \\
\hline & & 4 & $72.09 \pm 2.00$ & 2.63 & 0.24 & & 2.20 & 2.45 \\
\hline & \multirow{2}{*}{4} & 3 & $71.49 \pm 4.14$ & 2.19 & 0.20 & \multirow{2}{*}{640} & 2.93 & 3.14 \\
\hline & & 4 & $72.32 \pm 2.03$ & 2.63 & 0.24 & & 2.93 & 3.18 \\
\hline \multirow{7}{*}{70} & \multirow{2}{*}{2} & 3 & $70.70 \pm 0.65$ & 3.38 & 0.31 & \multirow{2}{*}{320} & 1.47 & 1.79 \\
\hline & & 4 & $71.41 \pm 2.23$ & 4.12 & 0.38 & & 1.47 & 1.85 \\
\hline & \multirow{3}{*}{3} & 3 & $70.83 \pm 2.08$ & 3.38 & 0.31 & \multirow{2}{*}{480} & 2.20 & 2.52 \\
\hline & & 4 & $68.78 \pm 0.25$ & 4.12 & 0.38 & & 2.20 & 2.59 \\
\hline & & 2 & $69.29 \pm 1.65$ & 2.62 & 0.24 & \multirow{3}{*}{640} & 2.93 & 3.18 \\
\hline & \multirow{2}{*}{4} & 3 & $70.30 \pm 0.16$ & 3.38 & 0.31 & & 2.93 & 3.25 \\
\hline & & 4 & $73.41 \pm 0.21$ & 4.12 & 0.38 & & 2.93 & 3.32 \\
\hline \multirow{11}{*}{80} & \multirow{3}{*}{2} & 2 & $69.37 \pm 1.37$ & 4.09 & 0.37 & \multirow{3}{*}{320} & 1.47 & 1.85 \\
\hline & & 3 & $71.75 \pm 0.99$ & 5.25 & 0.48 & & 1.47 & 1.96 \\
\hline & & 4 & $71.41 \pm 0.19$ & 6.31 & 0.58 & & 1.47 & 2.05 \\
\hline & \multirow{4}{*}{3} & 1 & $70.03 \pm 0.52$ & 2.88 & 0.26 & \multirow{4}{*}{480} & 2.20 & 2.47 \\
\hline & & 2 & $68.57 \pm 0.49$ & 4.09 & 0.37 & & 2.20 & 2.58 \\
\hline & & 3 & $71.77 \pm 0.35$ & 5.25 & 0.48 & & 2.20 & 2.69 \\
\hline & & 4 & $71.16 \pm 0.03$ & 6.31 & 0.58 & & 2.20 & 2.79 \\
\hline & \multirow{4}{*}{4} & 1 & $68.63 \pm 1.25$ & 2.88 & 0.26 & \multirow{4}{*}{640} & 2.93 & 3.21 \\
\hline & & 2 & $70.18 \pm 0.93$ & 4.09 & 0.37 & & 2.93 & 3.32 \\
\hline & & 3 & $69.66 \pm 0.65$ & 5.25 & 0.48 & & 2.93 & 3.42 \\
\hline & & 4 & $71.59 \pm 0.63$ & 6.31 & 0.58 & & 2.93 & 3.52 \\
\hline
\end{tabular}

${ }^{\mathrm{a}}$ based on pretreatment of $1.6 \mathrm{~kg}$ sugarcane bagasse with solid-liquid ratio of 1:10 (w:v), water consumption for all conditions is 0.0092 US dollar. 
Figures

\section{Fig. 1}

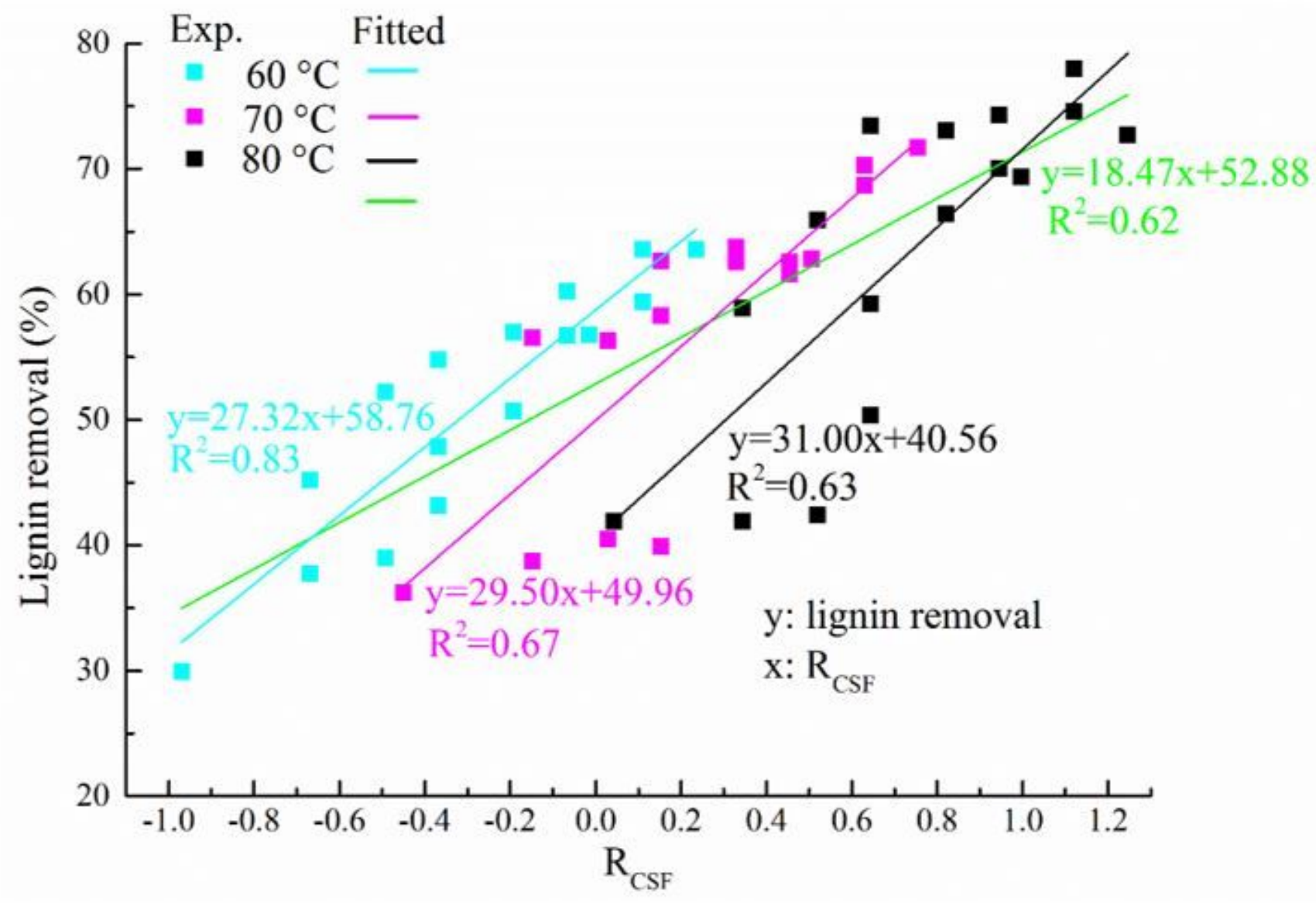

Figure 1

Correlation between the combined severity factor (RCSF) and lignin removal, Exp is short for experimental data, Fitted is short for fitted data, and green line fits the whole data obtained under all conditions 
Fig. 2
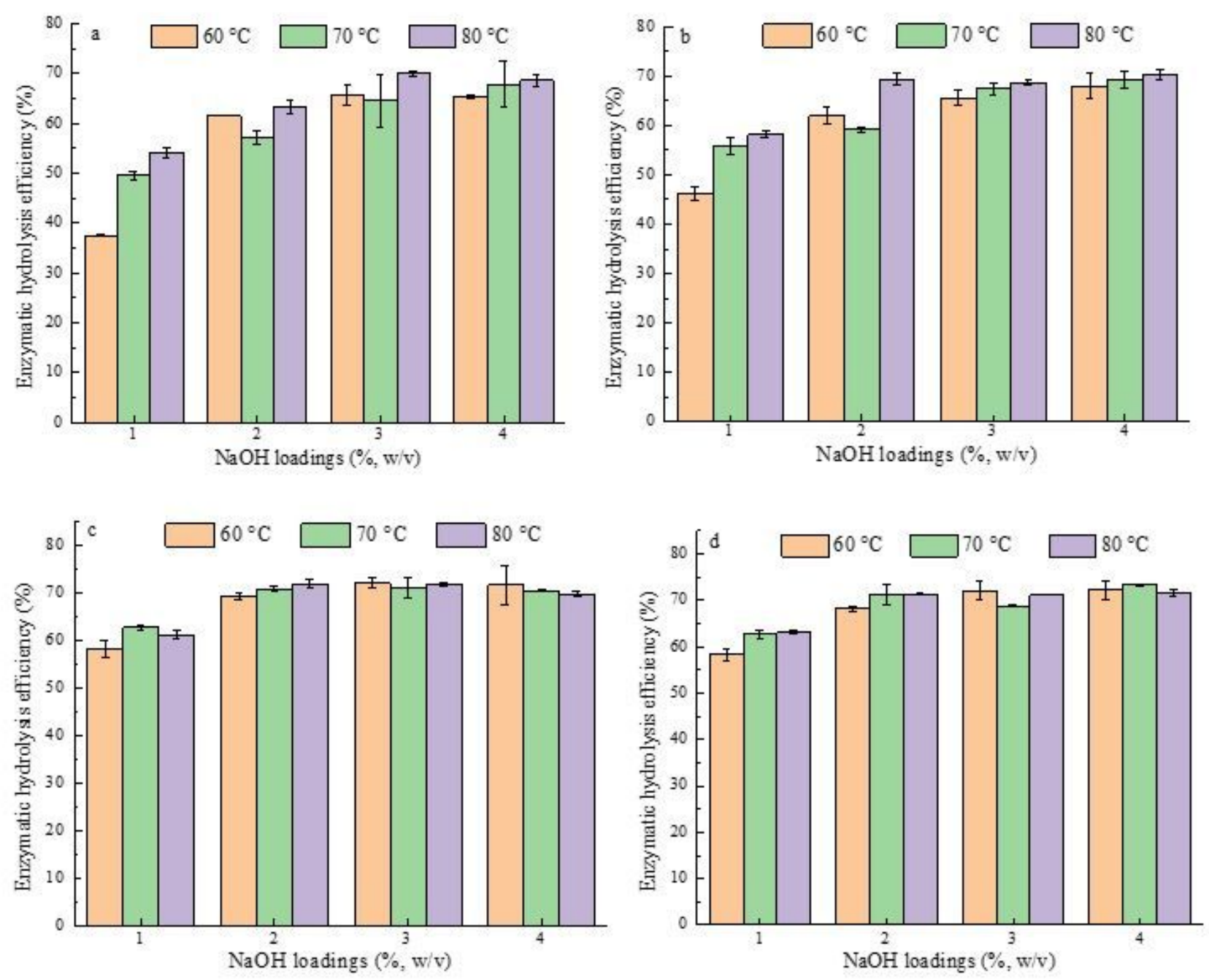

Figure 2

Enzymatic hydrolysis of SCB treated by $\mathrm{NaOH}$ solutions with different concentrations at $60^{\circ} \mathrm{C}, 70^{\circ} \mathrm{C}$ and $80^{\circ} \mathrm{C}$ for different times, (a) $1 \mathrm{~h}$, (b) $2 \mathrm{~h}$, (c) $3 \mathrm{~h}$, (d) $4 \mathrm{~h}$. The enzymatic hydrolysis was conducted under the condition of solid-liquid ratio of $1: 10$, cellulase loading of $20 \mathrm{FPU} / \mathrm{g}$ cellulose, $150 \mathrm{rpm}, 50^{\circ} \mathrm{C}$ for $72 \mathrm{~h}$ 


\section{Fig. 3}

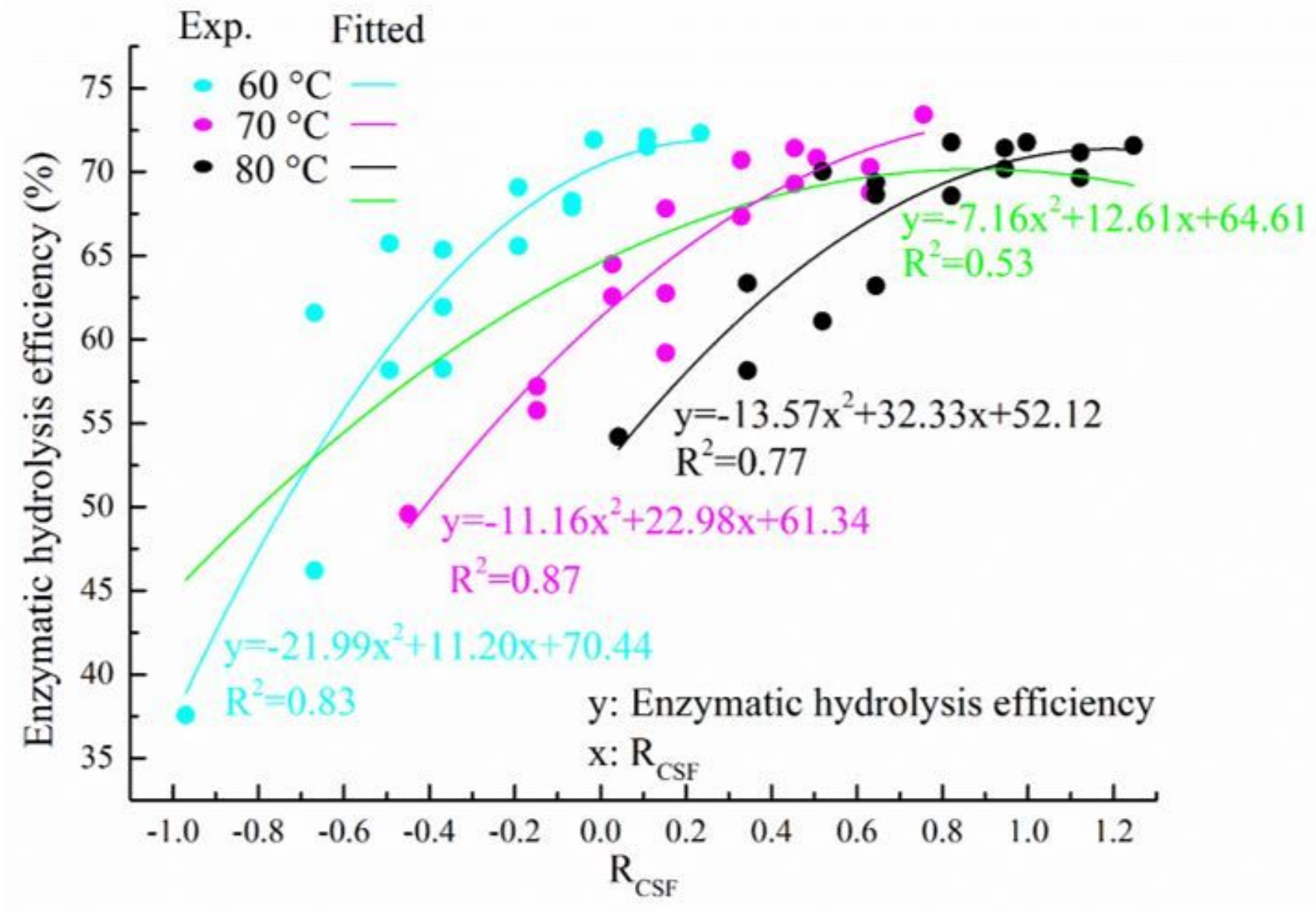

Figure 3

Correlation between the combined severity factor (RCSF) and enzymatic hydrolysis efficiency, Exp is short for experimental data, Fitted is short for fitted data, and green line fits the whole data obtained under all conditions 